Supporting Information for

\title{
Long Range Magnetic Ordering in Iron Jarosites Prepared by Redox-Based Hydrothermal Methods
}

\author{
Bart M. Bartlett and Daniel G. Nocera* \\ Department of Chemistry, 6-335, Massachusetts Institute of Technology, 77 Massachusetts \\ Avenue, Cambridge, MA 02139-4307
}

Index

Page

Figure S1. IR spectra of sulfate- and selenate-capped iron jarosites.

Figure S2. Powder X-ray diffraction pattern of plumbojarosite.

Figure S3. Zero-field cooled susceptibilities of sulfate- and selenate-capped iron jarosites.

Figure S4. $\left.(\mathrm{d} M / \mathrm{d} H)\right|_{\mathrm{T}}$ plots from which critical fields were determined for

$\mathrm{RbFe}_{3}(\mathrm{OH})_{6}\left(\mathrm{SO}_{4}\right)_{2}$ and $\mathrm{Pb}_{0.5} \mathrm{Fe}_{3}(\mathrm{OH})_{6}\left(\mathrm{SO}_{4}\right)_{2}$

$\mathrm{X}$-ray crystal structure data for $\mathrm{Pb}_{0.5} \mathrm{Fe}_{3}(\mathrm{OH})_{6}\left(\mathrm{SO}_{4}\right)_{2}$.

X-ray crystal structure data for $\mathrm{AgFe}_{3}(\mathrm{OH})_{6}\left(\mathrm{SO}_{4}\right)_{2}$.

X-ray crystal structure data for $\mathrm{TlFe}_{3}(\mathrm{OH})_{6}\left(\mathrm{SO}_{4}\right)_{2}$.

X-ray crystal structure data for $\mathrm{KFe}_{3}(\mathrm{OH})_{6}\left(\mathrm{SeO}_{4}\right)_{2}$.

X-ray crystal structure data for $\mathrm{RbFe}_{3}(\mathrm{OH})_{6}\left(\mathrm{SeO}_{4}\right)_{2}$. 


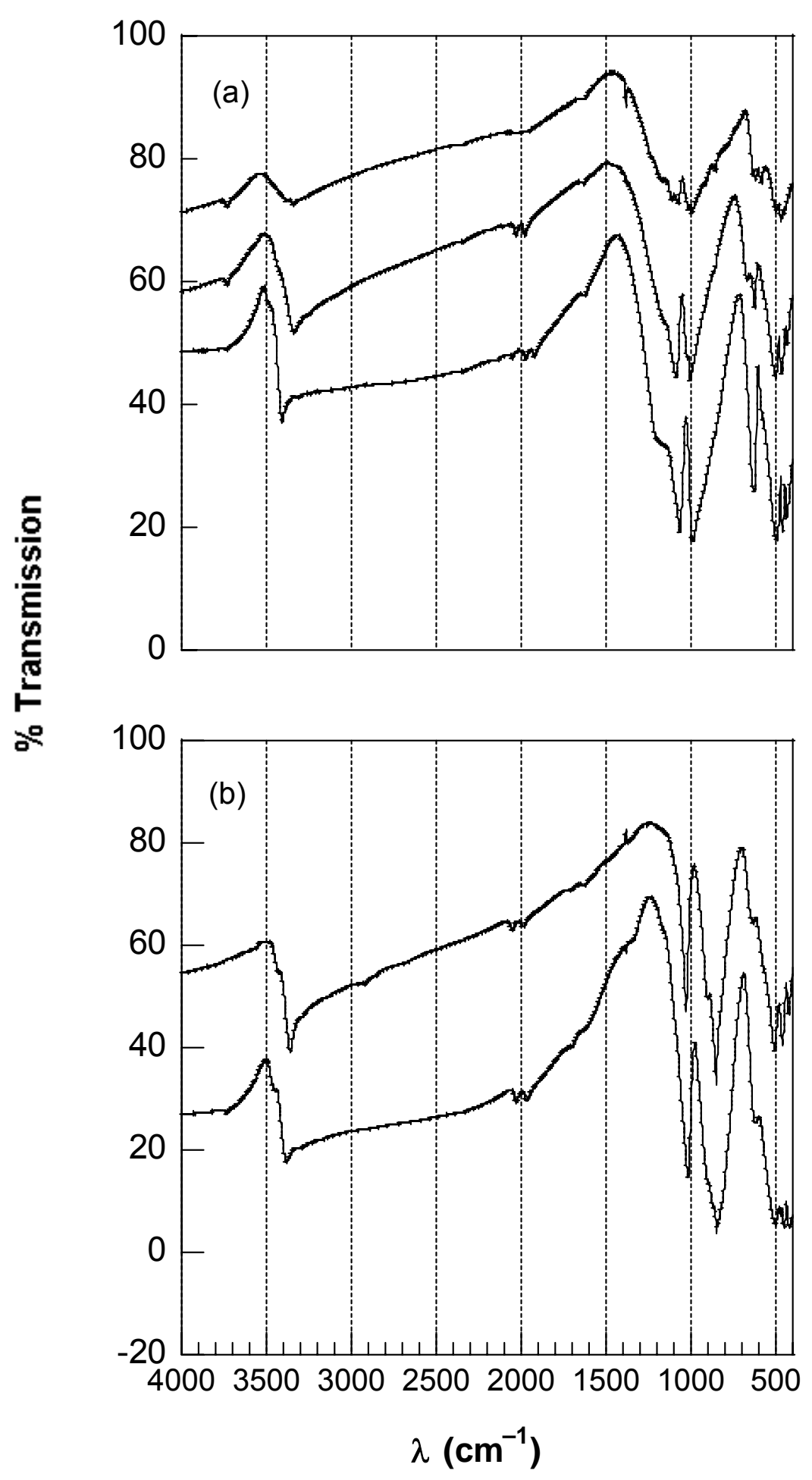

Figure S1. IR spectra of the (a) sulfate-capped iron jarosites, $\mathrm{Pb}_{0.5} \mathrm{Fe}_{3}(\mathrm{OH})_{6}\left(\mathrm{SO}_{4}\right)_{2}$ (top) $\mathrm{AgFe}_{3}(\mathrm{OH})_{6}\left(\mathrm{SO}_{4}\right)_{2}$ (middle), and $\mathrm{TlFe}_{3}(\mathrm{OH})_{6}\left(\mathrm{SO}_{4}\right)_{2}$ (bottom) and (b) selenate-capped iron jarosite analogs $\mathrm{KFe}_{3}(\mathrm{OH})_{6}\left(\mathrm{SO}_{4}\right)_{2}$ (top) and $\mathrm{RbFe}_{3}(\mathrm{OH})_{6}\left(\mathrm{SO}_{4}\right)_{2}$ (bottom). 


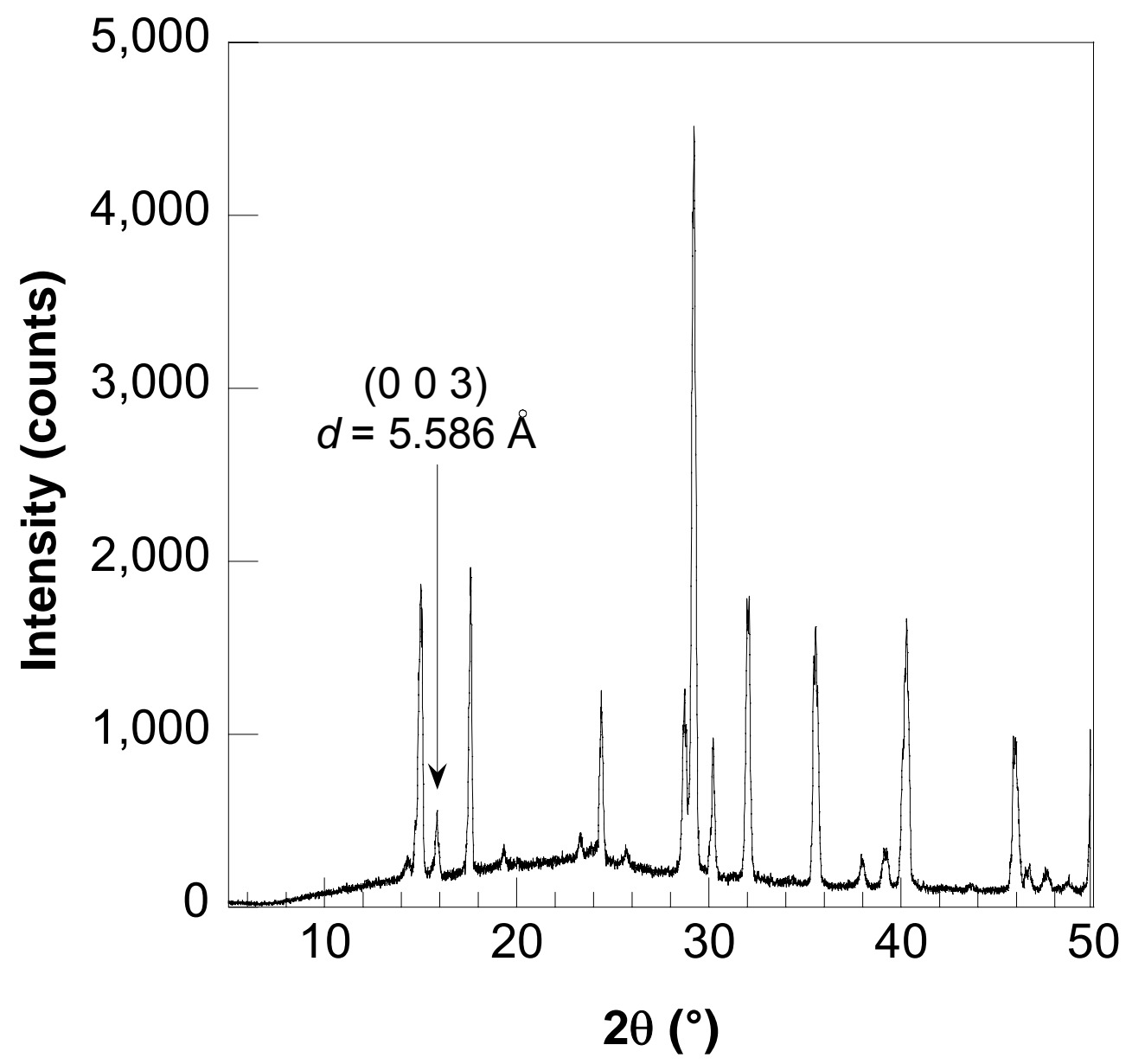

Figure S2. Powder X-ray diffraction pattern of $\mathrm{Pb}_{0.5} \mathrm{Fe}_{3}(\mathrm{OH})_{6}\left(\mathrm{SO}_{4}\right)_{2}$, emphasizing the $(003)$ reflection at $15.85^{\circ} 2 \theta$. 


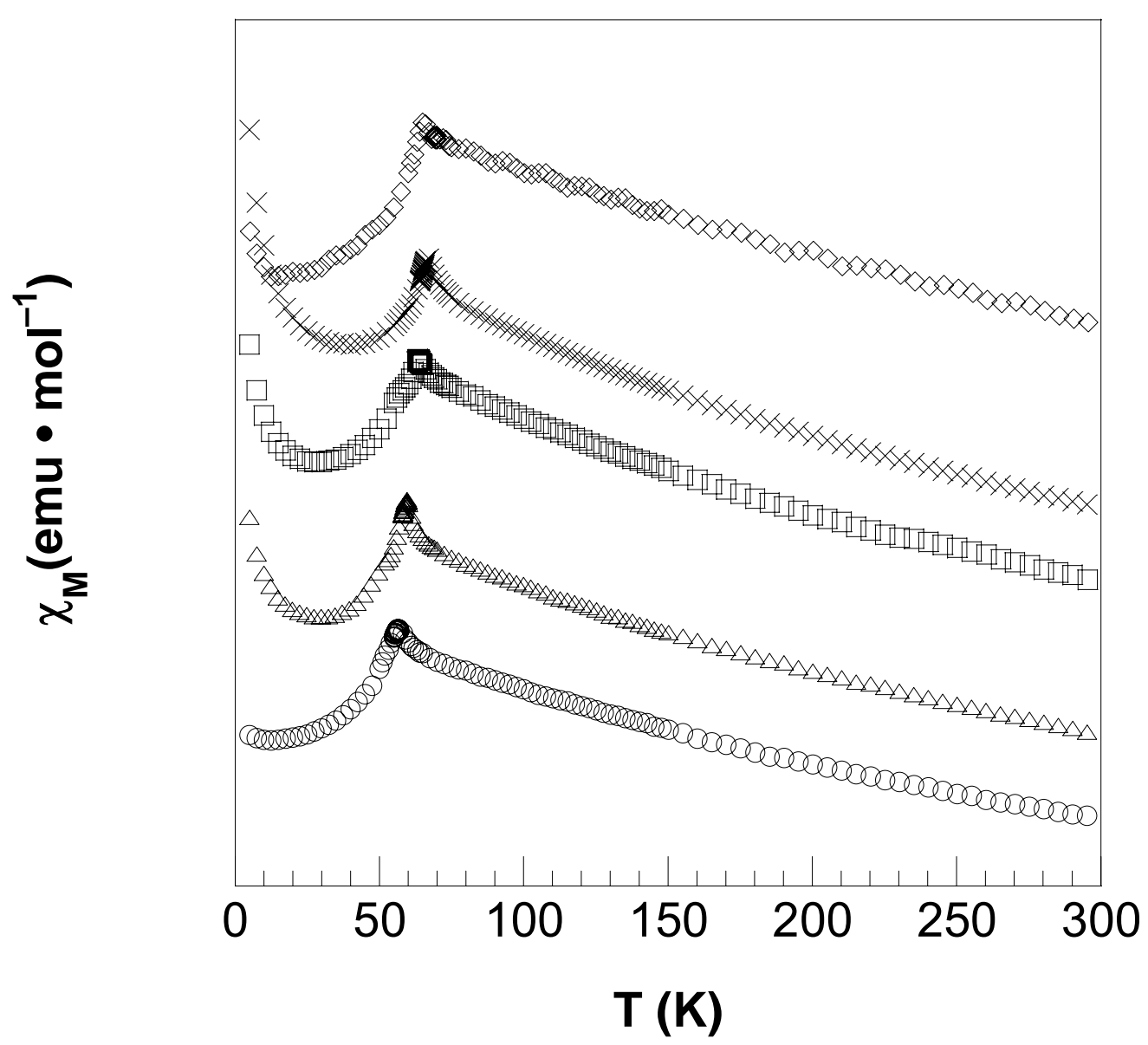

Figure S3. ZFC susceptibilities for the new jarosite compounds $\mathrm{Pb}_{0.5} \mathrm{Fe}_{3}(\mathrm{OH})_{6}\left(\mathrm{SO}_{4}\right)_{2}$ $(\circ), \mathrm{AgFe}_{3}(\mathrm{OH})_{6}\left(\mathrm{SO}_{4}\right)_{2} \quad(\Delta), \quad \mathrm{TlFe}_{3}(\mathrm{OH})_{6}\left(\mathrm{SO}_{4}\right)_{2} \quad(\square), \quad \mathrm{KFe}_{3}(\mathrm{OH})_{6}\left(\mathrm{SeO}_{4}\right)_{2} \quad(\times), \quad$ and $\mathrm{RbFe}_{3}(\mathrm{OH})_{6}\left(\mathrm{SeO}_{4}\right)_{2}(\diamond)$. The maximum in $T_{\mathrm{N}}$ ranges from $56.466 .5 \mathrm{~K}$. Plots are offset for clarity. 


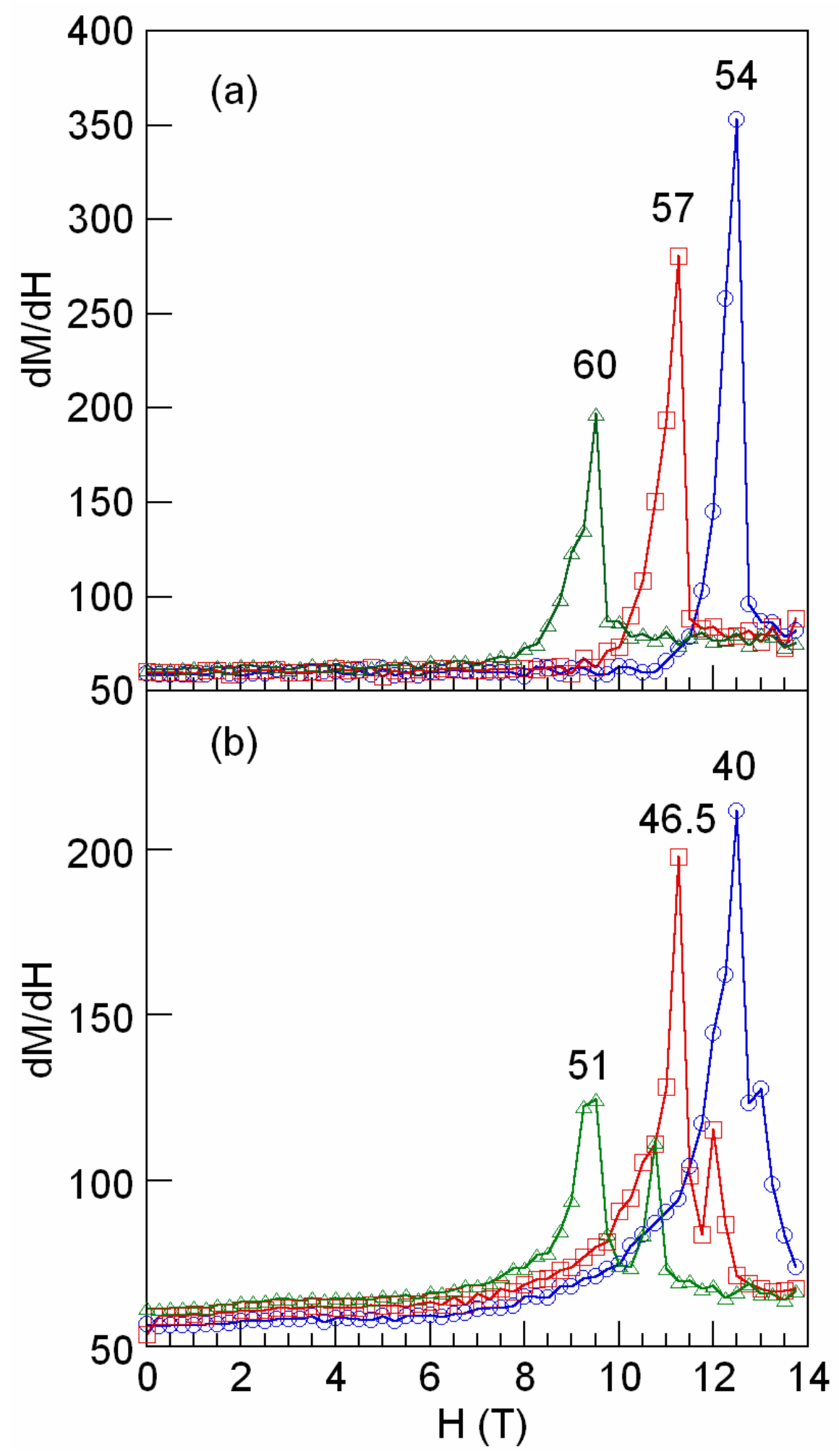

Figure S4. First derivative plots of the magnetization with applied field at a given temperature for (a) $\mathrm{RbFe}_{3}(\mathrm{OH})_{6}\left(\mathrm{SO}_{4}\right)_{2}$ and (b) $\mathrm{Pb}_{0.5} \mathrm{Fe}_{3}(\mathrm{OH})_{6}\left(\mathrm{SO}_{4}\right)_{2}$. The maximum gives the critical field for the ferromagnetic alignment of canted spins between layers. 


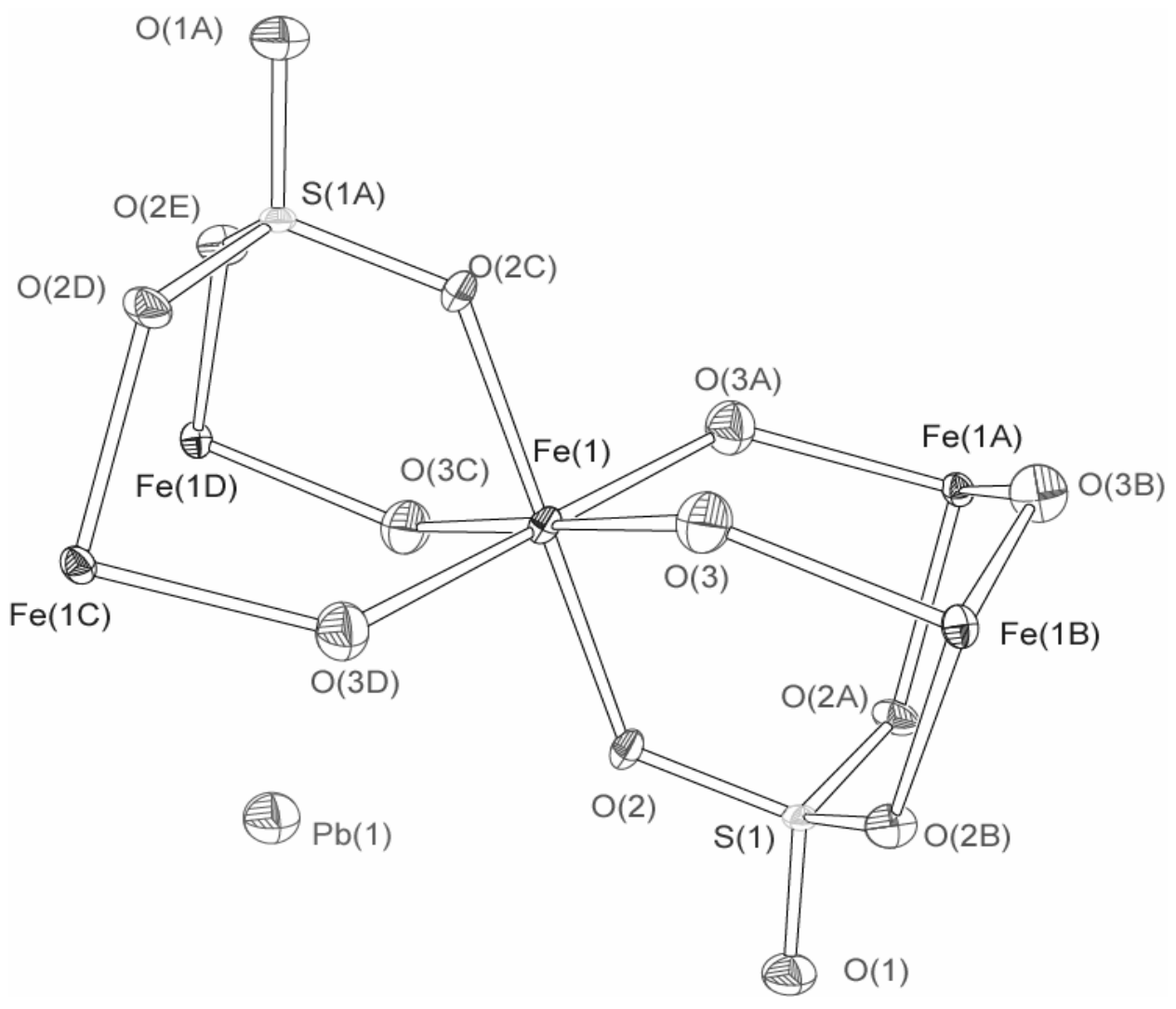

Figure S5. Atom labeling scheme for $\mathrm{Pb}_{0.5} \mathrm{Fe}_{3}(\mathrm{OH})_{6}\left(\mathrm{SO}_{4}\right)_{2}$. 
Table S1. Crystal data and structure refinement for $\mathrm{Pb}_{0.5} \mathrm{Fe}_{3}(\mathrm{OH})_{6}\left(\mathrm{SO}_{4}\right)_{2}$.

\begin{tabular}{|c|c|c|}
\hline Identification code & $001206 \mathrm{~m}$ & \\
\hline Empirical formula & $\mathrm{H}_{6} \mathrm{Fe}_{3} \mathrm{O}_{14} \mathrm{~Pb}_{0.5} \mathrm{~S}_{2}$ & \\
\hline Formula weight & 565.31 & \\
\hline Temperature & $183(2) \mathrm{K}$ & \\
\hline Wavelength & $0.71073 \AA$ & \\
\hline Crystal system & Rhombohedral & \\
\hline Space group & $R-3 \mathrm{~m}$ & \\
\hline \multirow[t]{3}{*}{ Unit cell dimensions } & $a=7.328(2) \AA$ & $\alpha=90^{\circ}$ \\
\hline & $b=7.328(2) \AA$ & $\beta=90^{\circ}$ \\
\hline & $c=16.795(6) \AA$ & $\gamma=120^{\circ}$ \\
\hline Volume & $781.1(4) \AA^{3}$ & \\
\hline $\mathrm{Z}$ & 3 & \\
\hline Density (calculated) & $3.606 \mathrm{Mg} / \mathrm{m}^{3}$ & \\
\hline Absorption coefficient & $15.334 \mathrm{~mm}^{-1}$ & \\
\hline $\mathrm{F}(000)$ & 848 & \\
\hline Crystal size & $0.04 \times 0.04 \times 0.04 \mathrm{~mm}^{3}$ & \\
\hline Theta range for data collection & 3.43 to $23.22^{\circ}$ & \\
\hline \multirow[t]{3}{*}{ Index ranges } & $-4 \leq h \leq 8$ & \\
\hline & $-8 \leq k \leq 5$ & \\
\hline & $-18 \leq l \leq 17$ & \\
\hline Reflections collected & 937 & \\
\hline Independent reflections & $164\left[\mathrm{R}\left({ }_{\mathrm{int}}\right)=0.0833\right]$ & \\
\hline Completeness to theta $=23.22^{\circ}$ & $100.0 \%$ & \\
\hline Absorption correction & None & \\
\hline Refinement method & Full-matrix least-squares on $\mathrm{F}^{2}$ & \\
\hline Data / restraints / parameters & $164 / 1 / 34$ & \\
\hline Goodness-of-fit on $\mathrm{F}^{2}$ & 1.190 & \\
\hline Final $R$ indices $[\mathrm{I}>2 \sigma(\mathrm{I})]$ & $R 1=0.0268$ & $w R 2=0.0538$ \\
\hline $\mathrm{R}$ indices (all data) & $R 1=0.0297$ & $w R 2=0.0552$ \\
\hline Largest diff. peak and hole & $0.736 \mathrm{e} \AA^{-3}$ & -0.593 e $\AA^{-3}$ \\
\hline
\end{tabular}


Table S2. Atomic coordinates $\left(\times 10^{4}\right)$ and equivalent isotropic displacement parameters $\left(\AA^{2} \times\right.$ $10^{3}$ ) for $\mathrm{Pb}_{0.5} \mathrm{Fe}_{3}(\mathrm{OH})_{6}\left(\mathrm{SO}_{4}\right)_{2}$. U(eq) is defined as one third of the trace of the orthogonalized $\mathrm{U}_{\mathrm{ij}}$ tensor.

\begin{tabular}{ccccc}
\hline Atom & $x$ & $y$ & $z$ & $\mathrm{U}(\mathrm{eq})$ \\
\hline $\mathrm{Pb}(1)$ & 0 & 0 & 0 & $21(1)$ \\
$\mathrm{S}(1)$ & 0 & 0 & $3109(2)$ & $7(1)$ \\
$\mathrm{Fe}(1)$ & 3333 & 1667 & 1667 & $8(1)$ \\
$\mathrm{O}(1)$ & 0 & 0 & $3973(4)$ & $20(3)$ \\
$\mathrm{O}(2)$ & $2200(7)$ & $1100(3)$ & $2810(2)$ & $15(2)$ \\
$\mathrm{O}(3)$ & $1266(3)$ & $2532(7)$ & $1343(2)$ & $18(2)$ \\
\hline
\end{tabular}

Table S3. Anisotropic displacement parameters $\left(\AA^{2} \times 10^{3}\right)$ for $\mathrm{Pb}_{0.5} \mathrm{Fe}_{3}(\mathrm{OH})_{6}\left(\mathrm{SO}_{4}\right)_{2}$. The anisotropic displacement factor exponent takes the form: $-2 \pi^{2}\left[h^{2} a^{* 2} U_{11}+\ldots+2 h k a * b * U_{12}\right]$

\begin{tabular}{lrrrllr}
\hline & $\mathrm{U}_{11}$ & $\mathrm{U}_{22}$ & $\mathrm{U}_{33}$ & $\mathrm{U}_{23}$ & $\mathrm{U}_{13}$ & $\mathrm{U}_{12}$ \\
\hline $\mathrm{Pb}(1)$ & $22(1)$ & $22(1)$ & $20(1)$ & 0 & 0 & $11(1)$ \\
$\mathrm{S}(1)$ & $8(1)$ & $8(1)$ & $5(1)$ & 0 & 0 & $4(1)$ \\
$\mathrm{Fe}(1)$ & $7(1)$ & $8(1)$ & $10(1)$ & $1(1)$ & $2(1)$ & $4(1)$ \\
$\mathrm{O}(1)$ & $22(4)$ & $22(4)$ & $14(4)$ & 0 & 0 & $11(2)$ \\
$\mathrm{O}(3)$ & $16(2)$ & $16(3)$ & $22(2)$ & $3(2)$ & $1(1)$ & $8(1)$ \\
$\mathrm{O}(2)$ & $10(3)$ & $19(2)$ & $13(2)$ & $1(1)$ & $3(2)$ & $5(1)$ \\
\hline
\end{tabular}

Table S4. Hydrogen coordinates $\left(\times 10^{4}\right)$ and isotropic displacement parameters $\left(\AA^{2} \times 10^{3}\right)$ for $\mathrm{Pb}_{0.5} \mathrm{Fe}_{3}(\mathrm{OH})_{6}\left(\mathrm{SO}_{4}\right)_{2}$.

\begin{tabular}{ccccc}
\hline & $x$ & $y$ & $z$ & $\mathrm{U}(\mathrm{eq})$ \\
\hline $\mathrm{H}(1)$ & $1920(40)$ & $3840(70)$ & $1050(40)$ & $60(50)$ \\
\hline
\end{tabular}




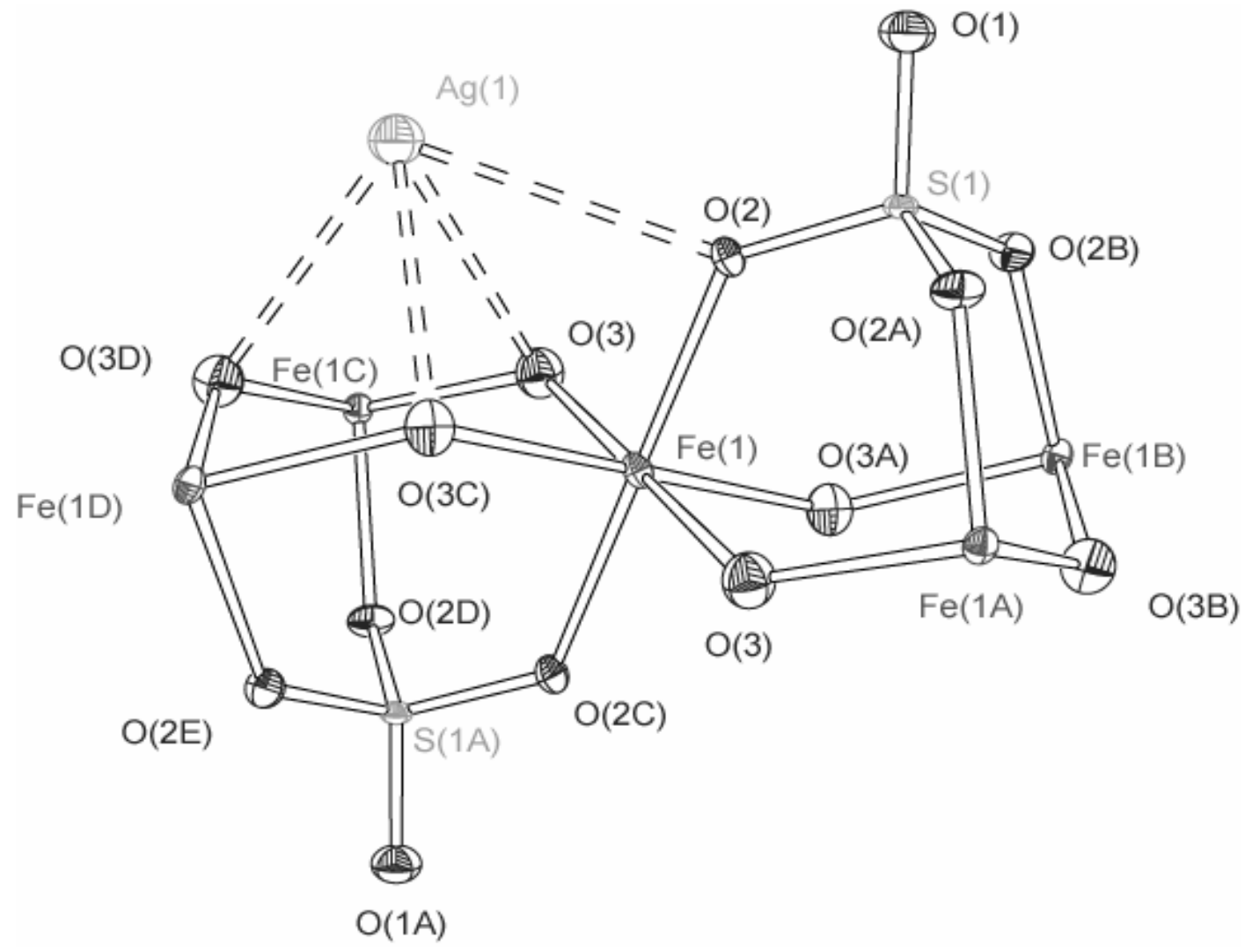

Figure S6. Atom labeling scheme for $\mathrm{AgFe}_{3}(\mathrm{OH})_{6}\left(\mathrm{SO}_{4}\right)_{2}$. 
Table S5. Crystal data and structure refinement for $\mathrm{AgFe}_{3}(\mathrm{OH})_{6}\left(\mathrm{SO}_{4}\right)_{2}$.

\begin{tabular}{|c|c|c|}
\hline Identification code & $002032 \mathrm{~m}$ & \\
\hline Empirical formula & $\mathrm{H}_{6} \mathrm{Fe}_{3} \mathrm{O}_{14} \mathrm{AgS}_{2}$ & \\
\hline Formula weight & 569.59 & \\
\hline Temperature & $183(2) \mathrm{K}$ & \\
\hline Wavelength & $0.71073 \AA$ & \\
\hline Crystal system & Rhombohedral & \\
\hline Space group & $R-3 \mathrm{~m}$ & \\
\hline \multirow[t]{3}{*}{ Unit cell dimensions } & $a=7.3300(9) \AA$ & $\alpha=90^{\circ}$ \\
\hline & $b=7.3300(9) \AA$ & $\beta=90^{\circ}$ \\
\hline & $c=16.497(3) \AA$ & $\gamma=120^{\circ}$ \\
\hline Volume & $767.62(19) \AA^{3}$ & \\
\hline $\mathrm{Z}$ & 3 & \\
\hline Density (calculated) & $3.696 \mathrm{Mg} / \mathrm{m}^{3}$ & \\
\hline Absorption coefficient & $6.547 \mathrm{~mm}^{-1}$ & \\
\hline $\mathrm{F}(000)$ & 825 & \\
\hline Crystal size & $0.04 \times 0.04 \times 0.04 \mathrm{~mm}^{3}$ & \\
\hline Theta range for data collection & 3.44 to $23.27^{\circ}$. & \\
\hline \multirow[t]{3}{*}{ Index ranges } & $-8 \leq h \leq 8$ & \\
\hline & $-8 \leq k \leq 8$ & \\
\hline & $-7 \leq l \leq 18$ & \\
\hline Reflections collected & 1039 & \\
\hline Independent reflections & $161[\mathrm{R}(\mathrm{int})=0.0459]$ & \\
\hline Completeness to theta $=23.27^{\circ}$ & $100.0 \%$ & \\
\hline Absorption correction & None & \\
\hline Refinement method & Full-matrix least-squares on $\mathrm{F}^{2}$ & \\
\hline Data / restraints / parameters & $161 / 1 / 29$ & \\
\hline Goodness-of-fit on $\mathrm{F}^{2}$ & 1.224 & \\
\hline Final $R$ indices $[\mathrm{I}>2 \sigma(\mathrm{I})]$ & $R 1=0.0255$ & $w R 2=0.0642$ \\
\hline $\mathrm{R}$ indices (all data) & $R 1=0.0259$ & $w R 2=0.0644$ \\
\hline Largest diff. peak and hole & $0.508 \mathrm{e}^{-3}$ & $-0.906 \mathrm{e} \AA^{-3}$ \\
\hline
\end{tabular}


Table S6. Atomic coordinates $\left(\times 10^{4}\right)$ and equivalent isotropic displacement parameters $\left(\AA^{2} \times\right.$ $10^{3}$ ) for $\mathrm{AgFe}_{3}(\mathrm{OH})_{6}\left(\mathrm{SO}_{4}\right)_{2}$. U(eq) is defined as one third of the trace of the orthogonalized $\mathrm{U}_{\mathrm{ij}}$ tensor.

\begin{tabular}{ccccc}
\hline Atom & $x$ & $y$ & $z$ & $\mathrm{U}(\mathrm{eq})$ \\
\hline $\mathrm{Ag}(1)$ & 0 & 0 & 0 & $27(1)$ \\
$\mathrm{S}(1)$ & 0 & 0 & $3123(2)$ & $7(1)$ \\
$\mathrm{Fe}(1)$ & 3333 & 1667 & 1667 & $7(1)$ \\
$\mathrm{O}(1)$ & 0 & 0 & $4009(5)$ & $8(2)$ \\
$\mathrm{O}(2)$ & $2194(8)$ & $1097(4)$ & $2823(3)$ & $10(1)$ \\
$\mathrm{O}(3)$ & $1256(4)$ & $2512(8)$ & $1331(3)$ & $8(1)$ \\
\hline
\end{tabular}

Table S7. Anisotropic displacement parameters $\left(\AA^{2} \times 10^{3}\right)$ for $\mathrm{AgFe}_{3}(\mathrm{OH})_{6}\left(\mathrm{SO}_{4}\right)_{2}$. The anisotropic displacement factor exponent takes the form: $-2 \pi^{2}\left[\mathrm{~h}^{2} \mathrm{a}{ }^{2} \mathrm{U}_{11}+\ldots+2 \mathrm{hka} \mathrm{b}^{*} \mathrm{U}_{12}\right]$.

\begin{tabular}{lrrrllr}
\hline & $\mathrm{U}_{11}$ & $\mathrm{U}_{22}$ & $\mathrm{U}_{33}$ & $\mathrm{U}_{23}$ & $\mathrm{U}_{13}$ & $\mathrm{U}_{12}$ \\
\hline $\mathrm{Ag}(1)$ & $34(1)$ & $34(1)$ & $13(1)$ & 0 & 0 & $17(1)$ \\
$\mathrm{S}(1)$ & $8(1)$ & $8(1)$ & $5(2)$ & 0 & 0 & $4(1)$ \\
$\mathrm{Fe}(1)$ & $5(1)$ & $7(1)$ & $8(1)$ & $0(1)$ & $1(1)$ & $3(1)$ \\
$\mathrm{O}(1)$ & $12(3)$ & $12(3)$ & $1(4)$ & 0 & 0 & $6(2)$ \\
$\mathrm{O}(2)$ & $6(3)$ & $13(2)$ & $7(2)$ & $0(1)$ & $0(2)$ & $3(1)$ \\
$\mathrm{O}(3)$ & $7(2)$ & $7(3)$ & $11(2)$ & $1(2)$ & $1(1)$ & $4(2)$ \\
\hline
\end{tabular}

Table S8. Hydrogen coordinates $\left(\times 10^{4}\right)$ and isotropic displacement parameters $\left(\AA^{2} \times 10^{3}\right)$ for $\mathrm{AgFe}_{3}(\mathrm{OH})_{6}\left(\mathrm{SO}_{4}\right)_{2}$.

\begin{tabular}{ccccc}
\hline & $x$ & $y$ & $z$ & $\mathrm{U}(\mathrm{eq})$ \\
\hline $\mathrm{H}(1)$ & $2000(20)$ & $3990(50)$ & $1180(70)$ & $120(70)$ \\
\hline
\end{tabular}




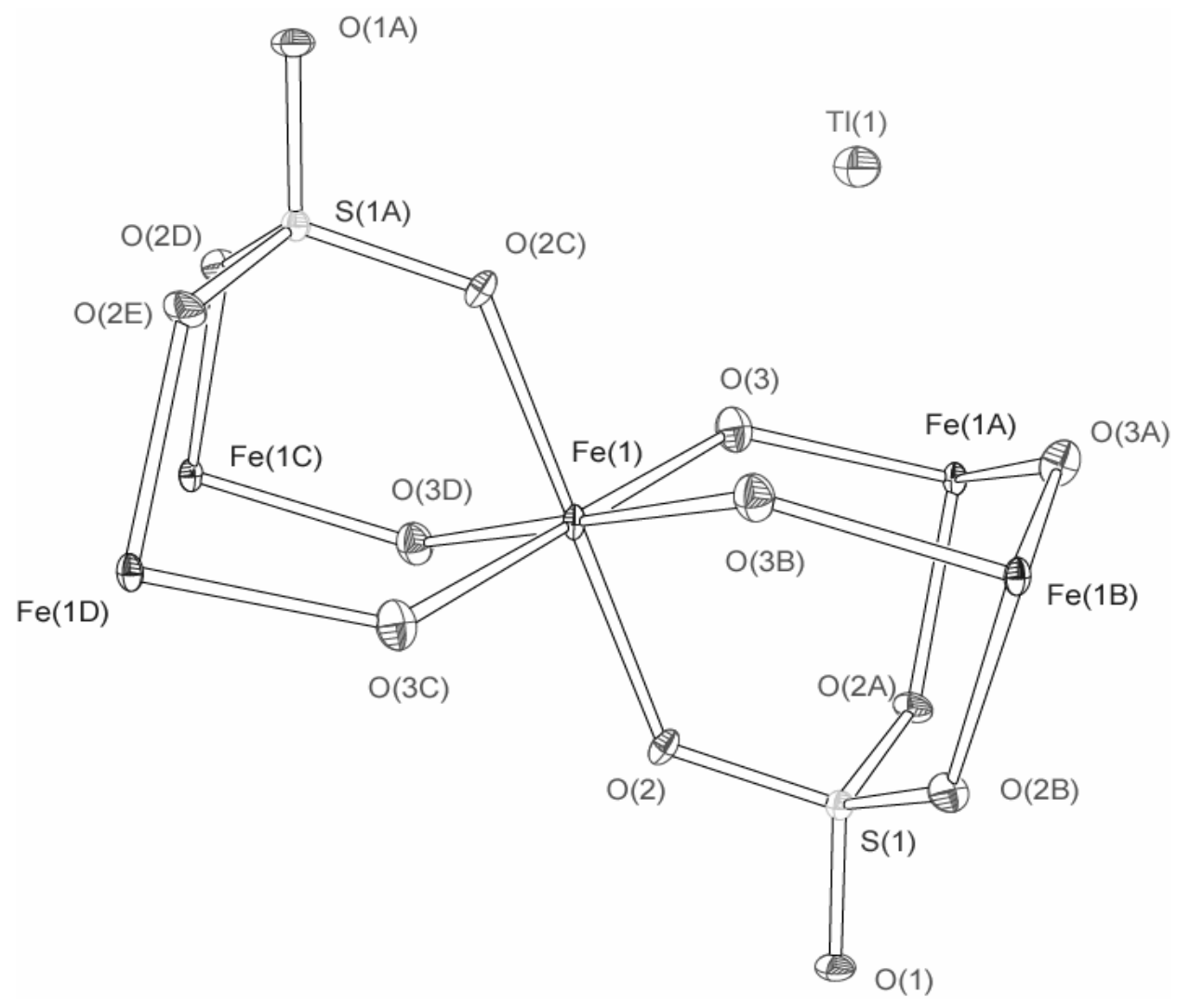

Figure S7. Atom labeling scheme for $\mathrm{TlFe}_{3}(\mathrm{OH})_{6}\left(\mathrm{SO}_{4}\right)_{2}$. 
Table S9. Crystal data and structure refinement for $\mathrm{TlFe}_{3}(\mathrm{OH})_{6}\left(\mathrm{SO}_{4}\right)_{2}$.

\begin{tabular}{lll}
\hline Identification code & $002124 \mathrm{~m}$ & \\
Empirical formula & $\mathrm{H}_{6} \mathrm{Fe}_{3} \mathrm{O}_{14} \mathrm{TlS}_{2}$ & \\
Formula weight & 666.09 & \\
Temperature & $183(2) \mathrm{K}$ & \\
Wavelength & $0.71073 \AA$ & \\
Crystal system & Rhombohedral & \\
Space group & $R-3 \mathrm{~m}$ & $\alpha=90^{\circ}$ \\
Unit cell dimensions & $a=7.3226(7) \AA$ & $\beta=90^{\circ}$ \\
& $b=7.3226(7) \AA$ & $\gamma=120^{\circ}$ \\
& $c=17.610(2) \AA$ & \\
Volume & $817.74(15) \AA^{3}$ & \\
$Z$ & 3 & \\
Density (calculated) & $4.058 \mathrm{Mg} / \mathrm{m}^{3}$ & \\
Absorption coefficient & $19.111 \mathrm{~mm}^{-1}$ & \\
F(000) & 927 & \\
Crystal size & $0.04 \times 0.04 \times 0.04 \mathrm{~mm}^{3}$ & \\
Theta range for data collection & 3.41 to $23.22^{\circ}$. & \\
Index ranges & $-6 \leq h \leq 8$ & \\
& $-7 \leq k \leq 8$ & \\
& $-18 \leq l \leq 19$ & \\
Reflections collected & 1080 & \\
Independent reflections & $169[\mathrm{R}($ int $)=0.0589]$ & \\
Completeness to theta $=23.22^{\circ}$ & $100.0 \%$ & \\
Absorption correction & None & \\
Refinement method & $\mathrm{Full-matrix} \mathrm{least-squares} \mathrm{on} \mathrm{F}^{2}$ & \\
Data / restraints / parameters & $169 / 1 / 29$ & \\
Goodness-of-fit on $\mathrm{F}^{2}$ & 1.292 & \\
Final R indices [I $>2 \sigma(\mathrm{I})]$ & $R 1=0.0207646$ \\
$\mathrm{R}$ indices (all data) & $R 1=0.0207$ & \\
Largest diff. peak and hole & $0.754 \mathrm{e} \AA^{-3}$ & \\
\hline
\end{tabular}


Table S10. Atomic coordinates $\left(\times 10^{4}\right)$ and equivalent isotropic displacement parameters $\left(\AA^{2} \times\right.$ $10^{3}$ ) for $\mathrm{TlFe}_{3}(\mathrm{OH})_{6}\left(\mathrm{SO}_{4}\right)_{2}$. U(eq) is defined as one third of the trace of the orthogonalized $\mathrm{U}_{\mathrm{ij}}$ tensor.

\begin{tabular}{ccccc}
\hline Atom & $x$ & $y$ & $z$ & $\mathrm{U}(\mathrm{eq})$ \\
\hline $\mathrm{Tl}(1)$ & 0 & 0 & 0 & $12(1)$ \\
$\mathrm{S}(1)$ & 0 & 0 & $3050(2)$ & $6(1)$ \\
$\mathrm{Fe}(1)$ & 3333 & 1667 & 1667 & $5(1)$ \\
$\mathrm{O}(1)$ & 0 & 0 & $3873(6)$ & $9(2)$ \\
$\mathrm{O}(2)$ & $2202(10)$ & $1101(5)$ & $2762(3)$ & $9(1)$ \\
$\mathrm{O}(3)$ & $1283(5)$ & $2565(10)$ & $1374(3)$ & $12(2)$ \\
\hline
\end{tabular}

Table S11. Anisotropic displacement parameters $\left(\AA^{2} \times 10^{3}\right)$ for $\mathrm{TlFe}_{3}(\mathrm{OH})_{6}\left(\mathrm{SO}_{4}\right)_{2}$. The anisotropic displacement factor exponent takes the form: $-2 \pi^{2}\left[h^{2} a^{* 2} U_{11}+\ldots+2 h k a * b * U_{12}\right]$.

\begin{tabular}{lrrrlll}
\hline & $\mathrm{U}_{11}$ & $\mathrm{U}_{22}$ & $\mathrm{U}_{33}$ & $\mathrm{U}_{23}$ & $\mathrm{U}_{13}$ & $\mathrm{U}_{12}$ \\
\hline $\mathrm{Tl}(1)$ & $13(1)$ & $13(1)$ & $11(1)$ & 0 & 0 & $7(1)$ \\
$\mathrm{S}(1)$ & $5(1)$ & $5(1)$ & $7(2)$ & 0 & 0 & $3(1)$ \\
$\mathrm{Fe}(1)$ & $3(1)$ & $4(1)$ & $9(1)$ & $0(1)$ & $0(1)$ & $2(1)$ \\
$\mathrm{O}(1)$ & $11(4)$ & $11(4)$ & $5(5)$ & 0 & 0 & $6(2)$ \\
$\mathrm{O}(2)$ & $6(3)$ & $11(2)$ & $9(3)$ & $2(1)$ & $3(3)$ & $3(2)$ \\
$\mathrm{O}(3)$ & $9(3)$ & $10(4)$ & $16(4)$ & $-2(3)$ & $-1(1)$ & $5(2)$ \\
\hline
\end{tabular}

Table S12. Hydrogen coordinates $\left(\times 10^{4}\right)$ and isotropic displacement parameters $\left(\AA^{2} \times 10^{3}\right)$ for $\mathrm{TlFe}_{3}(\mathrm{OH})_{6}\left(\mathrm{SO}_{4}\right)_{2}$.

\begin{tabular}{ccccc}
\hline & $x$ & $y$ & $z$ & $\mathrm{U}(\mathrm{eq})$ \\
\hline $\mathrm{H}(1)$ & $1300(300)$ & $2700(500)$ & $824(15)$ & $230(160)$ \\
\hline
\end{tabular}




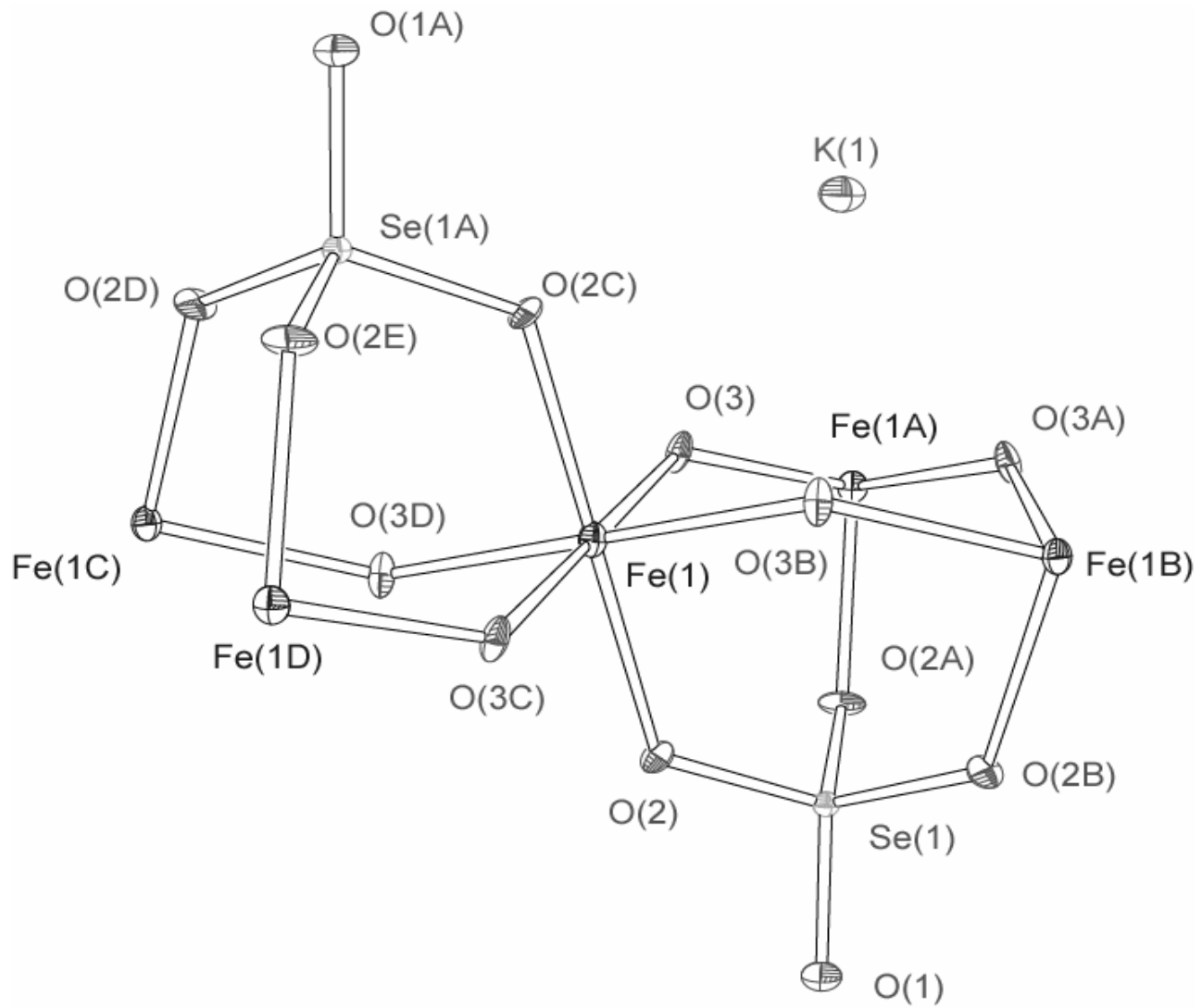

Figure S8. Atomic labeling scheme for $\mathrm{KFe}_{3}(\mathrm{OH})_{6}\left(\mathrm{SeO}_{4}\right)_{2}$. 
Table S13. Crystal data and structure refinement for $\mathrm{KFe}_{3}(\mathrm{OH})_{6}\left(\mathrm{SeO}_{4}\right)_{2}$.

\begin{tabular}{|c|c|c|}
\hline Identification code & $002127 \mathrm{~m}$ & \\
\hline Empirical formula & $\mathrm{H}_{6} \mathrm{Fe}_{3} \mathrm{O}_{14} \mathrm{KSe}_{2}$ & \\
\hline Formula weight & 594.62 & \\
\hline Temperature & $183(2) \mathrm{K}$ & \\
\hline Wavelength & $0.71073 \AA$ & \\
\hline Crystal system & Rhombohedral & \\
\hline Space group & $R-3 \mathrm{~m}$ & \\
\hline \multirow{3}{*}{ Unit cell dimensions } & $a=7.3902(9) \AA$ & $\alpha=90^{\circ}$ \\
\hline & $b=7.3902(9) \AA$ & $\beta=90^{\circ}$ \\
\hline & $c=17.498(3) \AA$ & $\gamma=120^{\circ}$ \\
\hline Volume & $827.6(2) \AA^{3}$ & \\
\hline $\mathrm{Z}$ & 3 & \\
\hline Density (calculated) & $3.579 \mathrm{Mg} / \mathrm{m}^{3}$ & \\
\hline Absorption coefficient & $10.947 \mathrm{~mm}^{-1}$ & \\
\hline $\mathrm{F}(000)$ & 849 & \\
\hline Crystal size & $0.04 \times 0.04 \times 0.02 \mathrm{~mm}^{3}$ & \\
\hline Theta range for data collection & 3.39 to $23.27^{\circ}$ & \\
\hline \multirow[t]{3}{*}{ Index ranges } & $-7 \leq h \leq 8$ & \\
\hline & $-7 \leq k \leq 8$ & \\
\hline & $-19 \leq l \leq 19$ & \\
\hline Reflections collected & 1130 & \\
\hline Independent reflections & $171\left[\mathrm{R}\left({ }_{\text {int }}\right)=0.0706\right]$ & \\
\hline Completeness to theta $=23.27^{\circ}$ & $100.0 \%$ & \\
\hline Absorption correction & None & \\
\hline Refinement method & Full-matrix least-squares on $\mathrm{F}^{2}$ & \\
\hline Data / restraints / parameters & $171 / 1 / 29$ & \\
\hline Goodness-of-fit on $\mathrm{F}^{2}$ & 1.245 & \\
\hline Final $R$ indices $[\mathrm{I}>2 \sigma(\mathrm{I})]$ & $R 1=0.0367$ & $w R 2=0.0781$ \\
\hline $\mathrm{R}$ indices (all data) & $R 1=0.0374$ & $w R 2=0.0786$ \\
\hline Largest diff. peak and hole & $0.913 \mathrm{e} \AA^{-3}$ & $-0.690 \mathrm{e} \AA^{-3}$ \\
\hline
\end{tabular}


Table S14. Atomic coordinates $\left(\times 10^{4}\right)$ and equivalent isotropic displacement parameters $\left(\AA^{2} \times\right.$ $10^{3}$ ) for $\mathrm{KFe}_{3}(\mathrm{OH})_{6}\left(\mathrm{SeO}_{4}\right)_{2}$. U(eq) is defined as one third of the trace of the orthogonalized $\mathrm{U}_{\mathrm{ij}}$ tensor.

\begin{tabular}{ccccr}
\hline Atom & $x$ & $y$ & $z$ & $\mathrm{U}(\mathrm{eq})$ \\
\hline $\mathrm{K}(1)$ & 0 & 0 & 0 & $13(1)$ \\
$\mathrm{Se}(1)$ & 0 & 0 & $3115(1)$ & $5(1)$ \\
$\mathrm{Fe}(1)$ & 3333 & 1667 & 1667 & $7(1)$ \\
$\mathrm{O}(1)$ & 0 & 0 & $4036(7)$ & $11(3)$ \\
$\mathrm{O}(2)$ & $2410(11)$ & $1205(6)$ & $2792(3)$ & $10(2)$ \\
$\mathrm{O}(3)$ & $1258(6)$ & $2516(12)$ & $1387(4)$ & $9(2)$ \\
\hline
\end{tabular}

Table S15. Anisotropic displacement parameters $\left(\AA^{2} \times 10^{3}\right)$ for $\mathrm{KFe}_{3}(\mathrm{OH})_{6}\left(\mathrm{SeO}_{4}\right)_{2}$. The anisotropic displacement factor exponent takes the form: $-2 \pi^{2}\left[h^{2} a^{* 2} U_{11}+\ldots+2 h k a * b * U_{12}\right]$.

\begin{tabular}{lrrrlll}
\hline & $\mathrm{U}_{11}$ & $\mathrm{U}_{22}$ & $\mathrm{U}_{33}$ & $\mathrm{U}_{23}$ & $\mathrm{U}_{13}$ & $\mathrm{U}_{12}$ \\
\hline $\mathrm{K}(1)$ & $15(2)$ & $15(2)$ & $10(3)$ & 0 & 0 & $7(1)$ \\
$\mathrm{Se}(1)$ & $5(1)$ & $5(1)$ & $5(1)$ & 0 & 0 & $3(1)$ \\
$\mathrm{Fe}(1)$ & $5(1)$ & $7(1)$ & $10(1)$ & $0(1)$ & $1(1)$ & $3(1)$ \\
$\mathrm{O}(1)$ & $12(5)$ & $12(5)$ & $7(6)$ & 0 & 0 & $6(2)$ \\
$\mathrm{O}(2)$ & $6(4)$ & $6(4)$ & $7(3)$ & $2(1)$ & $4(3)$ & $3(2)$ \\
$\mathrm{O}(3)$ & $5(3)$ & $6(4)$ & $16(4)$ & $4(3)$ & $2(2)$ & $3(2)$ \\
\hline
\end{tabular}

Table S16. Hydrogen coordinates $\left(\times 10^{4}\right)$ and isotropic displacement parameters $\left(\AA^{2} \times 10^{3}\right)$ for $\mathrm{KFe}_{3}(\mathrm{OH})_{6}\left(\mathrm{SeO}_{4}\right)_{2}$.

\begin{tabular}{ccccc}
\hline & $x$ & $y$ & $z$ & $\mathrm{U}(\mathrm{eq})$ \\
\hline $\mathrm{H}(1)$ & $1930(40)$ & $3860(80)$ & $1130(50)$ & $40(50)$ \\
\hline
\end{tabular}




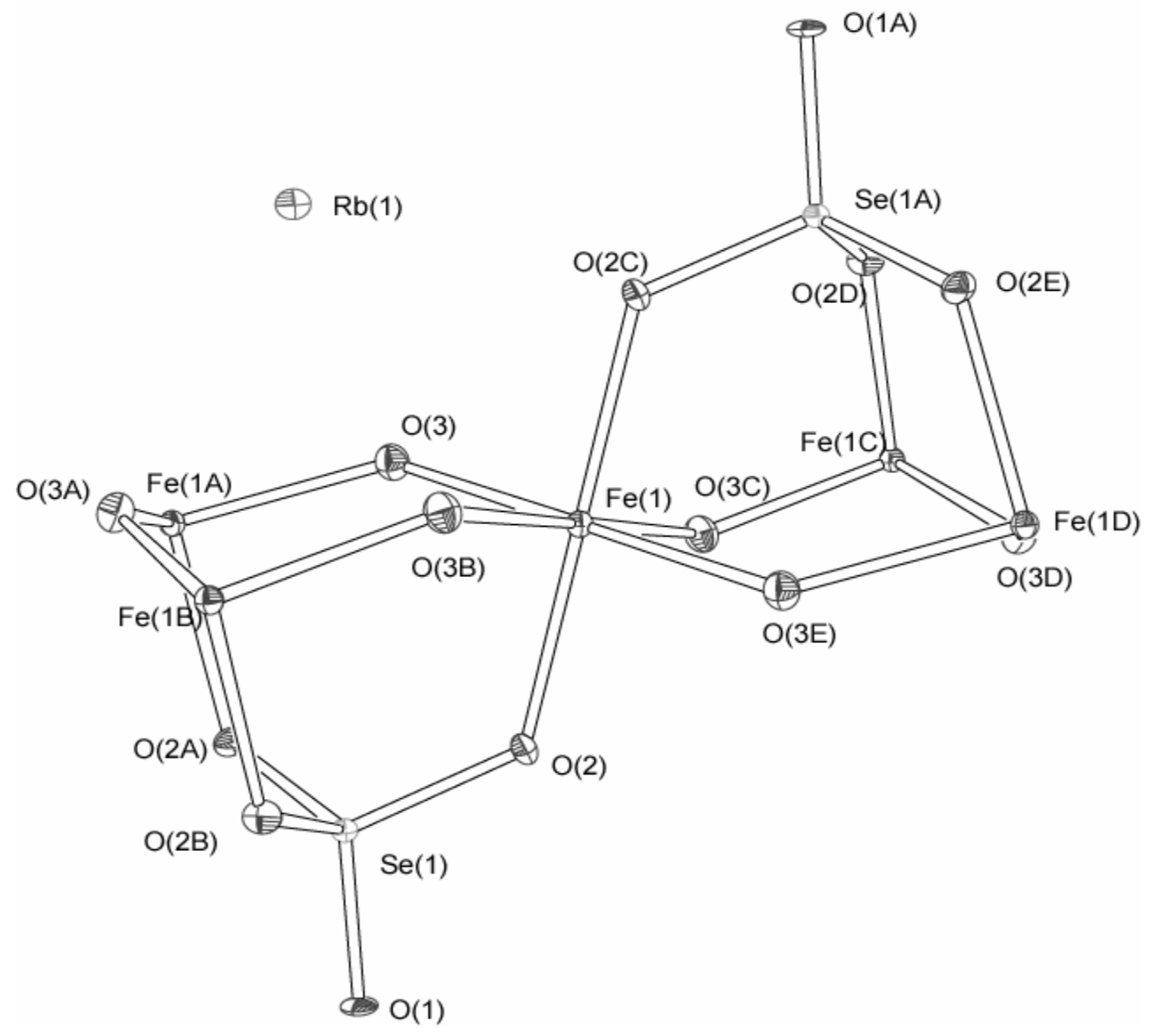

Figure S8. Atomic labeling scheme for $\mathrm{RbFe}_{3}(\mathrm{OH})_{6}\left(\mathrm{SeO}_{4}\right)_{2}$. 
Table S17. Crystal data and structure refinement for $\mathrm{RbFe}_{3}(\mathrm{OH})_{6}\left(\mathrm{SeO}_{4}\right)_{2}$.

\begin{tabular}{lll}
\hline Identification code & $003096 \mathrm{~m}$ & \\
Empirical formula & $\mathrm{H}_{6} \mathrm{Fe}_{3} \mathrm{O}_{14} \mathrm{RbSe}_{2}$ & \\
Formula weight & 640.99 & \\
Temperature & $100(2) \mathrm{K}$ & \\
Wavelength & $0.71073 \AA$ & \\
Crystal system & $\mathrm{Rhombohedral}$ & \\
Space group & $R-3 \mathrm{~m}$ & $\alpha=90^{\circ}$ \\
Unit cell dimensions & $a=7.4022(16) \AA$ & $\beta=90^{\circ}$ \\
& $b=7.4022(16) \AA$ & $\gamma=120^{\circ}$ \\
& $c=17.816(5) \AA$ & \\
Volume & $845.4(4) \AA^{3}$ & \\
Z & 3 & \\
Density (calculated) & $3.777 \mathrm{Mg} / \mathrm{m}^{3}$ & \\
Absorption coefficient & $4.879 \mathrm{~mm}^{-1}$ & \\
F(000) & 301 & \\
Crystal size & $0.04 \times 0.04 \times 0.03 \mathrm{~mm}^{3}$ & \\
Theta range for data collection & 3.38 to $28.47^{\circ}$. & \\
Index ranges & $-9 \leq h \leq 9$ & \\
& $-9 \leq k \leq 9$ & \\
& $-23 \leq l \leq 23$ & \\
Reflections collected & 5356 & \\
Independent reflections & $293[\mathrm{R}($ int $)=0.0323]$ & \\
Completeness to theta $=23.27^{\circ}$ & $100.0 \%$ & \\
Absorption correction & None & \\
Refinement method & $\mathrm{Full}-\mathrm{matrix}$ least-squares on $\mathrm{F}^{2}$ & \\
Data / restraints / parameters & $293 / 1 / 29$ & \\
Goodness-of-fit on $\mathrm{F}^{2}$ & 1.116 & \\
Final R indices [I $>2 \sigma(\mathrm{I})]$ & $R 1=0.01536$ \\
$\mathrm{R}$ indices (all data) & $R 1=0.0162$ & \\
Largest diff. peak and hole & $0.617 \mathrm{e} \AA^{-3}$ & \\
& & \\
\hline
\end{tabular}


Table S18. Atomic coordinates $\left(\times 10^{4}\right)$ and equivalent isotropic displacement parameters $\left(\AA^{2} \times\right.$ $10^{3}$ ) for $\mathrm{RbFe}_{3}(\mathrm{OH})_{6}\left(\mathrm{SeO}_{4}\right)_{2}$. U(eq) is defined as one third of the trace of the orthogonalized $\mathrm{U}_{\mathrm{ij}}$ tensor.

\begin{tabular}{ccccc}
\hline Atom & $x$ & $y$ & $z$ & $\mathrm{U}(\mathrm{eq})$ \\
\hline $\mathrm{Rb}(1)$ & 0 & 0 & 0 & $8(1)$ \\
$\mathrm{Se}(1)$ & 0 & 0 & $3093(1)$ & $5(1)$ \\
$\mathrm{Fe}(1)$ & 3333 & 1667 & 1667 & $5(1)$ \\
$\mathrm{O}(1)$ & 0 & 0 & $4002(2)$ & $8(1)$ \\
$\mathrm{O}(2)$ & $2409(3)$ & $1204(2)$ & $2774(1)$ & $8(1)$ \\
$\mathrm{O}(3)$ & $1264(2)$ & $2527(3)$ & $1408(1)$ & $10(1)$ \\
\hline
\end{tabular}

Table S19. Anisotropic displacement parameters $\left(\AA^{2} \times 10^{3}\right)$ for $\mathrm{RbFe}_{3}(\mathrm{OH})_{6}\left(\mathrm{SeO}_{4}\right)_{2}$. The anisotropic displacement factor exponent takes the form: $-2 \pi^{2}\left[\mathrm{~h}^{2} \mathrm{a}^{* 2} \mathrm{U}_{11}+\ldots+2 \mathrm{hka} \mathrm{b}^{*} \mathrm{U}_{12}\right]$.

\begin{tabular}{lrrrlll}
\hline & $\mathrm{U}_{11}$ & $\mathrm{U}_{22}$ & $\mathrm{U}_{33}$ & $\mathrm{U}_{23}$ & $\mathrm{U}_{13}$ & $\mathrm{U}_{12}$ \\
\hline $\mathrm{Rb}(1)$ & $8(1)$ & $8(1)$ & $7(1)$ & 0 & 0 & $4(1)$ \\
$\mathrm{Se}(1)$ & $5(1)$ & $5(1)$ & $5(1)$ & 0 & 0 & $3(1)$ \\
$\mathrm{Fe}(1)$ & $5(1)$ & $5(1)$ & $6(1)$ & $0(1)$ & $1(1)$ & $2(1)$ \\
$\mathrm{O}(1)$ & $10(1)$ & $10(10$ & $2(2)$ & 0 & 0 & $5(1)$ \\
$\mathrm{O}(2)$ & $5(1)$ & $10(1)$ & $8(1)$ & $1(1)$ & $1(1)$ & $2(1)$ \\
$\mathrm{O}(3)$ & $9(1)$ & $10(1)$ & $12(1)$ & $1(1)$ & $1(1)$ & $5(1)$ \\
\hline
\end{tabular}

Table S20. Hydrogen coordinates $\left(\times 10^{4}\right)$ and isotropic displacement parameters $\left(\AA^{2} \times 10^{3}\right)$ for $\mathrm{RbFe}_{3}(\mathrm{OH})_{6}\left(\mathrm{SeO}_{4}\right)_{2}$.

\begin{tabular}{ccccc}
\hline & $x$ & $y$ & $z$ & $\mathrm{U}(\mathrm{eq})$ \\
\hline $\mathrm{H}(1)$ & $1959(14)$ & $3920(30)$ & $1210(20)$ & $19(10)$ \\
\hline
\end{tabular}

